$\begin{gathered}\text { EPiC Series in Education Science } \\ \text { Volume 1, 2017, Pages 361-370 }\end{gathered}$
$\begin{aligned} & \text { AUBEA 2017: Australasian Universities Build- } \\ & \text { ing Education Association Conference 2017 }\end{aligned}$

\title{
A Change Management Perspective on BIM-FM Implementation
}

\author{
Julie Jupp ${ }^{1}$ and Ramsey Awad ${ }^{2}$ \\ ${ }^{1}$ University of Technology Sydney, Australia. \\ ${ }^{2}$ University of Newcastle, Australia. \\ julie.jupp@uts.edu.au, ramsey.awadenewcastle.edu.au
}

\begin{abstract}
Change plays a significant role in the implementation of any building information modelling (BIM) initiative. For owners transitioning from a traditional asset management $(\mathrm{AM})$ and/ or facilities management (FM) approach to one supported by BIM, change management is required due to the technological and organizational transformation involved. Yet little is known about the characteristics of change and how it can be more effectively managed. Based on a case study, this paper provides an example of a change strategy employed by a university's project delivery and operations teams to support the integration of BIM and FM. It describes a 'niche project' change management strategy and its key attributes during the early stages of a tertiary education owner/operator's transition to a model-based approach to asset and facilities management, providing a single point of truth for data storage and supporting 3D visualisation, digital work order processing and mobility in the field.
\end{abstract}

\section{Introduction}

Almost a decade ago, Eastman et al. (2008), defined as “.... a new approach to design, construction, and facilities management, in which a digital representation of the building process is used to facilitate the exchange and interoperability of information in digital format" (Eastman et al. 2008). Whilst BIM is a new approach to FM, comparatively low levels industry maturity have limited BIM's application to the project stages. However, an increasing number of research studies reflect the benefits, challenges and new technologies to facilitate the application of BIM to building operations. The aim of BIM-FM integration is to support operations by preventing the loss of building information, improving data access, and automating data entry. As a result, BIM-enabled FM can increase operational efficiency, reduce costs, increase the building lifecycle, and support collaboration and communication across business units. BIM-FM integration thus represents a major socio-technical challenge for the architectural, engineering, construction and operations (AECO) industry. 
A growing number of case studies describe the implementation and benefits of BIM-FM integration (Arayici et al. 2012, Kelly et al. 2013, Jupp 2013, Codinhoto \& Kiviniemi 2014, Kassem et al 2015, Jupp \& Awad 2017). Researchers have also begun to identify BIM-FM information requirements (Becerik-Gerber et al. 2012, Pinheiro et al. 2015, Ibrahim et al. 2016, Ashworth et al. 2016) and how they can be managed across the interfaces of the building lifecycle (Jupp \& Awad 2017, Jupp \& Singh 2016). What these studies highlight is the cross-functional nature of BIM-FM integration and its impact on the way an owner/operator runs their business, addresses their market, leverages core competences, and manages data.

This paper explores the key characteristics of change management that result from the implementation of a BIM-FM initiative. It focuses on change relative to the business context, technological and organizational issues of a university owner/operator organization. The paper presents the findings of a case study based on qualitative interviews with key personnel on a major university building project, and a review of project documents. The paper concludes with a discussion on alternative approaches to change management as owners and operators transition from traditional AM and FM methods to one supported by the digital flow of information using a 3D model-based approach as a single point of truth for data storage.

\section{Background}

Increasing levels of BIM maturity across design and construction activities are being documented in the literature, and project activities, which were once dispersed and siloed, are slowly being integrated via digital project delivery methods (Boton et al. 2017). With such changes to project delivery, opportunities for integrated, model-based approaches to information management in operations are beginning to be realized. Changes to AM and FM technologies, processes and protocols are growing.

\subsection{Transitioning from Traditional Operational Business Models}

The aim of any public and private owner/operator with multiple capital investments is "value creation', obtained by maximizing revenues while minimizing costs and inefficiencies in building operations and maintenance. Over recent years, value creation for owner/operators has transitioned from simple cost reduction to improving occupant experience and building performance (Jensen 2010). Consequently, a range of building services and maintenance activities are often outsourced and operations fragmented across specialist business units. Whilst outsourcing and specialization in building operations has increased the level of outward-facing 'openness', such business models can decrease internal collaboration and communication. An owner's development activities relating to project and program management are often therefore entirely separate from building services and maintenance activities, resulting in the dispersion of knowledge and information among different business units. As a result, owners have had to deal with siloes of knowledge and information.

Traditionally, FM teams have remained largely separate from project activities and the digital engineering competencies that have developed across the project delivery disciplines over the past decade. Compounding this problem, FM units must first undergo their own digital transformation with significant levels of integration required across existing tools and processes. Thus, support for a BIMFM initiative requires that owner/ operators undertake two key transformations, including the:

1. Transformation of information and communication technology (ICT) and underlying ICT infrastructure, allowing integration of all building related data via appropriate software.

2. Transformation of an interventional organizational view of the extended enterprise, enabling integration of AECO processes and activities across the entire building life cycle. 
The application of 'best of breed' technologies to support the use of BIM across AECO activities together with structured processes and supporting information management standards is resulting in the delivery of more accurate and well-coordinated geometric and non-geometric data. However in order to maximize the use, management and maintenance of buildings using a model-based approach and to minimize risk and operational costs, the occupancy phase together with AM and FM requirements must be considered from project inception. The recent development of Exchange Information Requirements (EIR) and the ISO/DIS 19650-1 standard was developed to support this objective. The ISO/DIS 196501 International Standard is primarily intended to support those involved in the procurement, design, construction and/or commissioning of built assets; as well as those involved in delivering asset management activities, including operations and maintenance. The specification of EIR across all client-side and project team stakeholders must therefore be undertaken early and continually verified and validated throughout project delivery phases (Ibrahim et al. 2016, Jupp \& Awad 2017).

Yet due to a lack of understanding of information requirement specification processes and competencies relating to information management across most operations stakeholders, the activities underpinning the execution of EIR activities are lacking in detail. Further, the use of quality control systems to ensure accurate and timely delivery of 3D model-based geometric and non-geometric data is also lacking in industry. Added to these missing links is the need for industry standards defining the processes and protocols for data harmonization and the connection of data and information collected during project delivery to AM and FM systems. This includes developing standards and greater levels of consistency in building classification schemas as well as labelling and naming conventions, which not only need to reflect operational AM and FM nomenclature of the asset owner but of particular sectors of construction and building types, e.g., hospitals, tertiary buildings, roads and bridges.

\subsection{Technological Transformation}

BIM-FM software is the 'connective tissue' that enables links between design, construction and FM processes throughout the enterprise. From this perspective, business operations can be divided into four streams: design management, supply management, construction management, and operations and service management. Each stream forms its own unique 'chain' of software, with interdependent information and data management requirements. From this software perspective, the chain of design processes are being supported via software such as 3D CAD (Computer Aided Design), CAWP (Computer Aided Workflow Planning) and CAPE (Computer Aided Manufacture). The chain of construction management processes is supported by tools such as QT (quantity take-off), 4D modelling (4D Planning and Scheduling), and 5D modelling (5D costing and resource management). Running across the design and construction management processes are a number of collaboration tools including EDM (Electronic Document Management) and CPM (Construction Project Management) systems).

Thus, the integration pathways of design and construction software are steadily maturing, with levels of interoperability supported by increasingly robust mappings between proprietary software and open standards such as the Industry Foundation Class (IFC) open standard (Liebich 2013). The IFC schema used together with buildingSMART's Information Delivery Manual (IDM) and Data Dictionary (ISO 2007, buildingSMART 2012) is capable of describing what kind of information can be exchanged by providing a mechanism that creates unique IFD IDs, thereby enabling a connection between information from existing and disparate databases to IFC data models (Laakso \& Kiviniemi 2012). A number of digital FM systems are currently available. However, the variety of systems employed by building owners often consists of ad-hoc combinations of 'off-the-shelf' FM and Building Management Systems (BMS) (Gökçe \& Gökçe 2013).

BIM-FM tool functionalities now commonly include five core capabilities: the capture of room data associated with the model (e.g., dRofus); interface support between BIM models and FM/asset management (e.g., Ecodomus); interface support between data sources and associated workflows (e.g., 
Zutec); support for building life cycle activities, from feasibility studies to design, construction and operation as well as FM/asset management via interoperable and transparent linkages between data (e.g., VEO M-six and VEO Archive); and audit processes for FM-ready models including maintenance and space data checking and data quality analysis (e.g., Invicara).

Further, firms pursuing enterprise wide integration and coupling project management ICTs with product data management (PDM) tools (e.g., Autodesk's Vault ${ }^{\mathrm{TM}}$ ) and Enterprise Resource Planning (ERP) solutions are increasing capabilities in and support for through-life information management. This enables design, construction operations and end user experience information to be linked and traced. The linking of information to the 3D model also provides the backdrop for data integration in PDM software to be employed (Holzer 2014). Whilst BIM-FM software integration and through-life approaches to information management across the AECO disciplines are therefore aspiring to more tightly coupled integration, there is currently no 'out of the box' product to provide a one-stop solution to integrate BIM with FM data. Existing approaches to achieving streamlined data transfer and management between software vary greatly.

Against this backdrop, the linking of BIM and FM software represents a recent development in industry and requires much needed development across all aspects of this information-centric approach if owner/ operators are to be able to implement it. A variety of AM and FM functions are currently supported by various BIM-FM software ranging from basic asset registry functions, managing defects, commissioning data, energy monitoring, emergency response, disaster planning, to maintenance scheduling (Becerik-Gerber et al. 2012). However, geometric and non-geometric data produced by design and construction teams is only useful if it is accurate, appropriately structured and formatted, and capable of linking to FM and/or the BMS.

Consequently, whilst AECO stakeholders are beginning the process of migrating their data storage and management from spreadsheet-based approaches to a centralized and integrated web-based platform, the actual increase in the information content and data management capabilities is currently constrained by the lack of consistent approaches to information structures and formats. The establishment and assignment of authoritative 'master' data in the chain of supporting workflows and modelling software, involves upfront planning and documentation (Holzer 2016). Consistency in the building classification schemas applied to the different building sectors is an example of the information management obstacles that must overcome if BIM-FM is to be implemented using a reliable approach. Model authoring tools, together with PDM and product lifecycle management (PLM) functionalities will therefore continue to inform AECO attempts to connect information through-life (Jupp 2016). From this technical point-of-view, BIM-FM requires the coordination and synthesis of three aspects of ICT, namely the:

(i) Consolidation of separate information systems across the owner's entire business into a centralized system for data storage and management, as well as software integration,

(ii) Awareness and planning of the tool ecologies used across AECO activities with detailed specification for data transfer and management, with an emphasis on local and remote collaboration tools using cloud-based technologies to support remote communication, and

(iii) Development of consistent processes and protocols that encompass both organizational and industry standards for information exchange and management.

\subsection{Organizational Transformation}

The availability of new BIM technologies allows for a substantial reshaping of an owner's business processes. This has a dramatic impact on an owner's internal organization. BIM-FM integration requires a close analysis of the way a building owner is engaging in design, construction and operations processes. That is how they specify and manage requirements, engage in design reviews, collaborate with project stakeholders, integrate processes and information, and collect, use and reuse data. 
Business process analysis techniques (Becker et al. 2013) should play a central role in any BIM-FM initiative. In fact, such an initiative requires careful understanding of business processes and the required process reengineering activities. More substantially than in project level deployments of BIM, implementing BIM for FM is a more technology intensive initiative, with even higher levels of organizational change required. Organizational change encompasses both processes and supporting protocols and therefore include changes in skills and competencies across the FM team.

Business process reengineering is therefore an essential process in the implementation of BIM-FM initiatives. Such an undertaking requires the analysis of existing procedures and work environments through "as-is" process analysis and the definition of 'to-be' processes. However, existing task analysis methodologies, such as unified modelling language, are arguably not well suited to this analysis, as they are unable to address required characteristics of BIM-FM integration. This is due to the main purpose of the methodologies being unable to include in their analysis and modelling BIM-FM attributes such as workflow and information. In addition, a careful equilibrium in the level of detail defined in the process analysis and documentation phase is required if it is to be useful.

Whilst an owner executes processes at a single point in time, flexible and adaptable solutions must be adopted where possible. The re-engineering phase has to be carried out in collaboration with relevant managers according to the objectives, and may require senior management involvement. For example, if a future 'to be' process includes the use of a model-based function that is expected to be disseminate across departments, it is necessary to establish agreement so as to support organizational change and related technical competency requirements. Communication and documentation of proposed changes to business processes are therefore key.

To assist in the analysis of the required organizational changes, collaborative workshops are being introduced. These techniques reflect experiential learning approaches (Kolb 1975) which allow stakeholders from different organizational departments identify shared knowledge and EIRs. In this way, experiential learning techniques can help owner organizations (and supporting AECO firms) to redefine the business processes that individuals are involved in through workshop sessions about new ways of working. Workshop enablers can include the use of EIR templates such as COBie (Construction Operations Building Information Exchange), providing a tool for participants to identify common ground. Virtual simulation of new operational activities can also be undertaken by teams to explore "to be' scenarios; allowing learning of new organizational needs. In this way, drawbacks are identified, allowing refinement of the 'to be' model.

After business process and operational process analyses are concluded - with the specification of EIRs - the next aspect of organizational change is to establish the sequence and protocols for software use across project phases and FM departments. With the rapid evolution of BIM-FM software tools, it is necessary to select those that support present and future needs. Therefore, particular attention should be paid not only to the internal road map established for the necessary digital transformations to FM strategy but also according to future development.

\subsection{Change Management Strategies}

Change management plays an essential and core part of any BIM-FM integration initiative. To support such transformations two strategies can be employed: the deployment of a 'niche project with follow up', or an 'overall step-by-step' approach. The first supports the definition of a niche application area inside the organization to introduce and verify the results and benefits of a proposed change. This approach looks at the transition-transformation process as if it were an experiment to be undertaken in a comparatively short timeframe. Motivated personnel are typically identified to support the niche project, and implementation timeframes are compressed. The goal is for tangible results to be identified from the project's execution and for lessons learned to be documented and used to secure further expansion of the initiative across the entire company. 
The second approach devotes much more time to the careful planning and process reengineering of the organization to include the full company. In this case, the definition of the 'as is' and 'to be' engineering process models play a fundamental role, as do workshops and carefully managed experiential learning sessions. Training is also carefully linked to process reengineering techniques under this change management strategy as they have to drive and control the overall step-by-step implementation. The latter strategy is less common as it is difficult to implement due to the upfront planning and resolution of numerous unknowns in EIRs and related workflows. The remainder of this paper explores the first of these strategies, the 'niche project with feedback' change management strategy.

\section{BIM-FM in the Tertiary Education Sector}

In Australia, tertiary education buildings are managed using a range of disparate software and are equipped with a variety of building automation systems. Any Australian university's ability to manage maintenance and operations data whilst monitoring performance or predicting maintenance is at best sporadic and at worst unreliable due to the varying levels of use and application across the campus. This ad-hoc combination presents difficulties for building owners in relation to the management and integration of existing AM and FM systems and the transformational changes that a model-based approach represents. Existing software lacks sufficient support for the exchange of information between applications; nor do current offerings consider the necessary extensions and in-field application devices of wired/ wireless monitoring or control systems for both operational staff and building users (Gökçe \& Gökçe 2013).

A case study focusing on the extraction of qualitative descriptions was used to gather data on BIMFM implementation processes in the tertiary education sector to support this research. The study describes the nature of a 'niche project' change management strategy during the deployment of a BIMFM integration initiative. The study was undertaken in collaboration with the owner-operator, an Australian university including the project management office (PMO) and facilities management unit (FMU), as well as the main contractor.

\subsection{Case Background}

The case studied describes a large university's initial BIM for FM project, which was based on a 'management contracting' procurement method. The building project comprised of a relatively small, four story clinical education facility. All design processes and some construction activities were supported by BIM and were collaborative from conception through to handover, involving both the PMO and FMU staff working with the entire project team. Four of the PMO and FMU participants, and two from the main contractor, were selected for interviews based on their level of participation in the project. Two sets of interviews were conducted with these participants. A number of project documents, including the BIM Execution Plan (BEP), BIM-FM Implementation Exchanges, BIM Contract Addendum and internal technical reports) were also reviewed.

The purpose of the interviews was to extract qualitative descriptions of change management techniques utilized during the niche project approach to BIM-FM integration. In terms of BIM or digital FM knowledge at the start of the project, the University was in a similar situation to many other organizations, in which there was a general internal consensus that the use of BIM should be adopted in both project procurement and FM, even if the details of what that actually meant were not yet fully understood. The University engaged a BIM consultancy firm to research and report to them how the organization could adopt a BIM based approach to FM. At the conclusion of the white paper it was decided that the new building project was to act as a pilot project trialing some of the papers recommendations. 
Thus, together with other members of the AECO project team delivering the building and the BIM consultant, some of the report's recommendations were put into action. As this was a niche project and the project team wanted to create something that was simple, easy to use, accessible, the agreed deliverable was a simple 3D as-built model accessed via a free model compilation viewer with embedded FM data attached to only specific maintainable building elements. The key processes that were undertaken to manage and deliver the FM ready BIM model and the BIM-FM integration system architecture were staged across the project timeline.

\subsection{Findings}

The main features of taking a 'niche project' approach are presented relative to three areas of BIMenabled FM, namely: (i) how technical and organizational requirements were identified, (ii) how sociotechnical changes were managed, and (iii) how communication on progress was conveyed.

(i) Requirements Identification: A BIM-FM working group was established and consisted of representatives from the university's project management office, the facilities management office, a bespoke data management team, a BIM consultancy and members from the main contractor. Meetings included interviews and presentations from the various stakeholders and interest groups. After several months, the working party was able to specify broadly the EIR and in doing so had to overcome four key challenges including: how to define a vision for using a BIM based FM solution; to what extent integration with existing FM software should occur; what AM and FM information should be captured and in what format, and where this information should be stored and accessed.

The search for solutions to address these challenges - including an industry standard and a software platform to support information management through-life - proved to be time intensive and was seen as a decision "too great for the assembled working group" on what was a standalone project. Therefore, the decision was made to keep the project's data in an open source format for partial integration with the University's existing FM software and employ an approach that allowed for integration into a BIMbased FM software tool at a later stage. To create a link between an element and the rest of its FM data, the main contractor used a set of asset names and IDs in conjunction with the Omniclass classification system to support search functions in the university's existing FM database where the bulk of the FM information for the project was to be stored. Throughout the working group process, the main contractor had a live draft of the BEP, which was continually updated allowing for the experiential learning to be captured in real time. The BEP was refined as the working group settled on data deliverables, workflows, data formats, etc. The owner, architect, contractor and subcontractors agreed to the final version of BEP.

(ii) Management of Socio-Technical Transformation: During this stage, the technical requirements of subcontractors were deployed and found to evolve or increase, meaning that the procurement process for them needed careful management. It was necessary to ensure that the main contractor was able to employ subcontractors who could understand and deliver according to the BEP and "buy into" why the accuracy of the data and as built information was especially important on this job. Although the technical aspects of the model were not onerous, there were small technical-related issues that some subcontractors had to overcome, such as ensuring good communication of workflows relating to model use, model coordination, and model management, enforcement of the BEP, and stakeholder management.

(iii) Progress Reporting: The quality and accuracy of as-built models will directly determine the usability and value of BIM data inputs relative to FM data outputs. Progress reporting during these data verification and harmonization processes were therefore critical. The outcomes of model audit and coordination activities were conveyed regularly to senior management. Documentation and management of model coordination workflows across all disciplines (and datasets) was therefore key to the initiative's success. Verification and validation of building service models against actual installation was undertaken; however, the use of laser scanning was seen to be potentially cost 
prohibitive on this scale of the project. It was therefore proposed that approximately 30 locations per floor, involving building services, be inspected or photographed and visually compared to the federated model. As an audit method, it was perceived to be effective as the photo only needed to indicate that there was a variance between the design and the installation at which point the subcontractor was instructed to coordinate and update the model. The following progress reporting requirements were identified as key:

- Ease of access to all geometric and non-geometric data contained in discipline models, where software interfaces had to be simple and free,

- Regular communication surrounding the specified tolerance and LOD of the as-built representation of physical assets, and

- Use of saved viewpoints for ease and speed of communication.

At building hand-over, the main contractor also established a feedback system so as to continue progress reporting and gather information on how the model was or was not being used by the FM team and how the data provide was being integrated..

\section{Discussion}

An appropriate coordination effort is required to focus and direct all technical and organisational development activities surrounding BIM-FM integration in the short to mid-term. After senior management commits to the project, a change management strategy has to pursue the following path:

(i) Confirm regular information to top management on project implementation steps,

(ii) Ensure regular communication on project progress to all involved, and

(iii) Apply experiential learning approaches in change management activities so as to secure understanding, buy-in and feedback.

With regard to these requirements, the advantages and disadvantages of a niche project approach are shown in Table 1

\begin{tabular}{|c|c|}
\hline Advantages & Disadvantages \\
\hline $\begin{array}{l}\text { Motivated and involved personnel are made } \\
\text { available }\end{array}$ & $\begin{array}{l}\text { Difficulty in feeding forward into the subsequent, } \\
\text { full scale, implementation }\end{array}$ \\
\hline Quick implementation of the technical solution & $\begin{array}{l}\text { Difficulty in evaluating the extent of project } \\
\text { achievements and scale them }\end{array}$ \\
\hline Focused resource allocation & Project is known only to a few persons \\
\hline Objective outcomes achieved in short term horizon & $\begin{array}{l}\text { Fewer variables to be controlled, which presents } \\
\text { additional risks in subsequent full scale } \\
\text { implementation }\end{array}$ \\
\hline
\end{tabular}

Table 1 Disadvantages and advantages in 'niche project approach'

BIM-FM integration relies on an owner organization's communication and coordination network to support the delivery of accurate as-built models and achieve the benefits of their use in operations. The more buildings in an owner's portfolio, the more a BIM-FM initiative will be complicated and require a clear strategy for change management. A global outlook on the BIM-FM systems architecture and its data processing system is therefore necessary. It is the goal of the information requirements, which enable BIM-FM integration to support the network of specialized software tools. Thus, information exchange protocols and information requirements management frameworks should be defined earlier by the owner, rather than be left to the project implementation team. 
The organizational change management path may be carried out progressively, project-by-project, or via a full-scale step-by-step pathway, according to senior management. The ability to support the correct use of BIM and FM tools will be an important criterion for making this decision. In order to benefit from the advantages of both solutions, mixed change management strategies involving both niche project and overall systematic approaches could provide a solution. This would enable 'as is' and 'to be' situations to be analyzed in full, in order to have a 'global project' that can be presented to senior management for approval. The combination of two different approaches may be most effective to support experiential learning in the first instance and push users to modify their attitudes towards new reengineered processes, before enabling the required software training and practical use afterwards.

\section{References}

Jensen, AP. (2010). The facilities management value map: a conceptual framework. Facilities, 28(3/4), 175-188.

Arayici, Y., Onyenobi T. \& Egbu, C (2012). Building information modelling (BIM) for facilities management (FM): The MediaCity case study approach. Intl. Journal of 3D Information Modeling (IJ3DIM) 1(1): 55-73.

Ashworth, S., Tucker, M., Druhmann, C., \& Kassem, M. (2016). Integration of FM expertise and end user needs in the BIM process using the Employer's Information Requirements. In Proceedings of CIB World Building Congress (Vol. 5).

Becker, J., Rosemann, M., \& Von Uthmann, C. (2000). Guidelines of business process modeling. In Business Process Management (pp. 30-49). Springer Berlin Heidelberg.

Becerik-Gerber, B., Jazizadeh, F., Li, N., \& Calis, G. (2012). Application areas and data requirements for BIM-enabled facilities management. Journal of construction engineering and management, 138(3), 431-442.

BSRIA (2009), The Soft Landings Framework, BSRIA.

buildingSMART (2012). IFD Library for buildingSMART 2012.

Boton C, Rivest, L, Forgues D \& Jupp JR (2017), A Comparison of Shipbuilding and Construction Industries from the Product Structure Standpoint, International Journal of Product Lifecycle Management, Inderscience Publishers.

Codinhoto, R., \& Kiviniemi, A. (2014). BIM-FM: A Case Support for Business Life Cycle. In IFIP Intl. Conf. on PLM (pp. 63-74). Springer Berlin Heidelberg.

Gökçe, H., \& Gökçe, K. (2013). Integrated system platform for energy efficient building operations. J. of Comp. in Civil Eng., 28(6), 05014005.

Holzer, D. (2014). Fostering the Link from PLM to ERP via BIM. In IFIP International Conference on Product Lifecycle Management (pp. 75-82). Springer Berlin Heidelberg.

Ibrahim, K. F., Abanda, F. H., Vidalakis, C., \& Woods, G. (2016) BIM-FM: Input versus Output data, Proc. of the 33rd CIB W78 Conference, Oct. 31st - Nov. 2nd, Bris., Australia.

ISO Standard. ISO 12006-3:2007 Building construction: Organization of information about construction works, Part 3: Framework for object-oriented information. 2007.

ISO Standard ISO/DIS 19650-1 Organization of information about construction works Information management using building information modelling

ISO Standard. ISO 16739:2013 Industry Foundation Classes (IFC) for data sharing in the construction and facility management industries, 2013.

Jupp JR. (2013). Incomplete BIM implementation: Exploring challenges and role of product lifecycle management functions, International Conference on Product Lifecycle Management (PLM 2013), 6-10 July, Nantes, France.

Jupp JR. (2016). Cross industry learning: a comparative study of product lifecycle management and building information modelling, Intl. Jrl of Product Lifecycle Management, Inderscience Publishers. 
Jupp JR. \& Awad R. (2017). BIM for FM and Information Requirements Management: Missing Links in the AEC and FM Interface, International Conference on Product Lifecycle Management (PLM 2017), 9-12 July, Seville, Spain.

Jupp JR \& Singh V: 2016, Advancing BIM as a Strategy Business Approach, International Journal of Product Lifecycle Management, Inderscience Publishers.

Kassem, M., Kelly, G., Dawood, N., Serginson, M., \& Lockley, S. (2015). BIM in facilities management applications: A case study of a large university complex. Built Environment Project and Asset Mgmt, 5(3), 261-277.

Kelly, G., Serginson, M., Lockley, S., Dawood, N., \& Kassem, M. (2013). BIM for facility management: A review and a case study investigating the value and challenges. In Proc. of the 13th Intl. Conf. on Construction Applications of Virtual Reality (pp. 30-31).

Kolb, D. A. (1975). Toward an applied theory of experiential learning. Theories of group processes.

Laakso, M. \& Kiviniemi, A., (2012). The IFC standard - A review of history, development, and standardization. ITcon, 17, pp.134-161.

Liebich, T., 2013. IFC4 -The New buildingSMART Standard: What's new in IFC4?

Pinheiro, S. V., Corry, E., \& O'Donnell, J. T. (2015). Requirements for a BIM-Based Life-Cycle Performance Evaluation Framework to Enable Optimum Building Operation. In 32nd Intl. CIB W78 Conf., Eindhoven, The Netherlands, 27-29 Oct. 2015 (pp. 639-648). EUT. 